Article

\title{
A Proof of a Conjecture on Bipartite Ramsey Numbers $B(2,2,3)$
}

\author{
Yaser Rowshan ${ }^{1}$, Mostafa Gholami ${ }^{1}$ and Stanford Shateyi ${ }^{2, * \mathbb{D}}$ \\ 1 Department of Mathematics, Institute for Advanced Studies in Basic Sciences (IASBS), \\ Zanjan 66731-45137, Iran; y.rowshan@iasbs.ac.ir (Y.R.); gholami.m@iasbs.ac.ir (M.G.) \\ 2 Department of Mathematics and Applied Mathematics, School of Mathematical and Natural Sciences, \\ University of Venda, P. Bag X5050, Thohoyandou 0950, South Africa \\ * Correspondence: stanford.shateyi@univen.ac.za
}

\begin{abstract}
The bipartite Ramsey number $B\left(n_{1}, n_{2}, \ldots, n_{t}\right)$ is the least positive integer $b$, such that any coloring of the edges of $K_{b, b}$ with $t$ colors will result in a monochromatic copy of $K_{n_{i}, n_{i}}$ in the $i$-th color, for some $i, 1 \leq i \leq t$. The values $B(2,5)=17, B(2,2,2,2)=19$ and $B(2,2,2)=11$ have been computed in several previously published papers. In this paper, we obtain the exact values of the bipartite Ramsey number $B(2,2,3)$. In particular, we prove the conjecture on $B(2,2,3)$ which was proposed in 2015-in fact, we prove that $B(2,2,3)=17$.
\end{abstract}

Keywords: Ramsey numbers; bipartite Ramsey numbers; Zarankiewicz number

Citation: Rowshan, Y.; Gholami, M.; Shateyi, S. A Proof of a Conjecture on Bipartite Ramsey Numbers $B(2,2,3)$. Mathematics 2022, 10, 701. https:// doi.org/10.3390/math10050701 Academic Editors: Janez Žerovnik and Darja Rupnik Poklukar

Received: 7 December 2021 Accepted: 23 December 2021 Published: 23 February 2022

Publisher's Note: MDPI stays neutral with regard to jurisdictional claims in published maps and institutional affiliations.

Copyright: (c) 2022 by the authors. Licensee MDPI, Basel, Switzerland. This article is an open access article distributed under the terms and conditions of the Creative Commons Attribution (CC BY) license (https:// creativecommons.org/licenses/by/ $4.0 /)$.

\section{Introduction}

The bipartite Ramsey number $B\left(n_{1}, n_{2}, \ldots, n_{t}\right)$ is the least positive integer $b$, such that any coloring of the edges of $K_{b, b}$ with $t$ colors will result in a monochromatic copy of $K_{n_{i}, n_{i}}$ in the $i$-th color, for some $i, 1 \leq i \leq t$. The existence of such a positive integer is guaranteed by a result of Erdős and Rado [1].

The Zarankiewicz number $z\left(K_{m, n}, t\right)$ is defined as the maximum number of edges in any subgraph $G$ of the complete bipartite graph $K_{m, n}$, such that $G$ does not contain $K_{t, t}$ as a subgraph. Zarankiewicz numbers and related extremal graphs have been studied by many authors, including Kóvari [2], Reiman [3], and Goddard, Henning, and Oellermann in [4].

The study of bipartite Ramsey numbers was initiated by Beineke and Schwenk in 1976 [5], and continued by others, in particular Exoo [6], Hattingh, and Henning [7]. The following exact values have been established: $B(2,5)=17$ [8], $B(2,2,2,2)=19$ [9], $B(2,2,2)=11$ [6]. In the smallest open case for five colors, it is known that $26 \leq B(2,2,2,2,2) \leq 28$ [9]. One can refer to [2,9-14] and it references for further studies. Collins et al. in [8] showed that $17 \leq B(2,2,3) \leq 18$, and in the same source made the following conjecture:

Conjecture 1 ([8]). $B(2,2,3)=17$

We intend to get the exact value of the multicolor bipartite Ramsey numbers $B(2,2,3)$. We prove the following result:

Theorem 1. $B(2,2,3)=17$.

In this paper, we are only concerned with undirected, simple, and finite graphs. We follow [15] for terminology and notations not defined here. Let $G$ be a graph with vertex set $V(G)$ and edge set $E(G)$. The degree of a vertex $v \in V(G)$ is denoted by $\operatorname{deg}_{G}(v)$, or simply by $\operatorname{deg}(v)$. The neighborhood $N_{G}(v)$ of a vertex $v$ is the set of all vertices of $G$ adjacent to $v$ and satisfies $\left|N_{G}(v)\right|=\operatorname{deg}_{G}(v)$. The minimum and maximum degrees of vertices of $G$ are denoted by $\delta(G)$ and $\Delta(G)$, respectively. Additionally, the complete bipartite graph with bipartition $(X, Y)$, where $|X|=m$ and $|Y|=n$, is denoted by $K_{m, n}$. We use $[X, Y]$ to denote the set of edges between the bipartition $(X, Y)$ of $G$. Let $G=(X, Y)$ be a bipartite graph 
and $Z \subseteq X$ or $Z \subseteq Y$, the degree sequence of $Z$ denoted by $D_{G}(Z)=\left(d_{1}, d_{2}, \ldots, d_{|Z|}\right)$, is the list of the degrees of all vertices of $Z$. The complement of a graph $G$, denoted by $\bar{G}$, is a graph with same vertices such that two distinct vertices of $\bar{G}$ are adjacent if and only if they are not adjacent in $\mathrm{G}$. $H$ is $n$-colorable to $\left(H_{1}, H_{2}, \ldots, H_{t}\right)$ if there exists a $t$-coloring of the edges of $H$ such that $H_{i} \nsubseteq H^{i}$ for each $1 \leq i \leq t$, where $H^{i}$ is the spanning subgraph of $H$ with edges of the $i$-th color.

\section{Some Preliminary Results}

To prove our main result-namely, Theorem 1-we need to establish some preliminary results. We begin with the following proposition:

Proposition 1 ([8,13]). The following results about the Zarankiewicz number are true:

- $\quad z\left(K_{17,17}, 2\right)=74$.

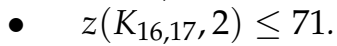

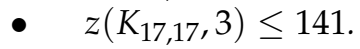

- $z\left(K_{16,17}, 3\right) \leq 133$.

- $z\left(K_{13,17}, 3\right) \leq 110$.

- $z\left(K_{12,17}, 3\right) \leq 103$.

- $\quad z\left(K_{11,17}, 3\right) \leq 96$.

Proof of Proposition 1. By using the bounds in Table 3 and Table 4 of [8] and Table C.3 of [13], the proposition holds.

Theorem 2 ([8]). $17 \leq B(2,2,3) \leq 18$.

Proof of Theorem 2. The lower bound witness is found in Table 2 of [8]. The upper bound is implied by using the bounds in Table 3 and Table 4 of [8]. We know that $z\left(K_{18,18}, 2\right)=81$, $z\left(K_{18,18}, 3\right) \leq 156$, and $2 \times 81+156=318<324=\left|E\left(K_{18,18}\right)\right|$.

Suppose that $\left(G^{r}, G^{b}, G^{g}\right)$ is a 3-edge coloring of $K_{17,17}$, where $K_{2,2} \nsubseteq G^{r}, K_{2,2} \nsubseteq G^{b}$ and $K_{3,3} \nsubseteq G^{g}$; in the following theorem, we specify some properties of the subgraph with color $g$. The properties are regarding $\Delta\left(G^{g}\right), \delta\left(G^{g}\right), E\left(G^{g}\right)$, and degree sequence of vertices $X, Y$ in the induced graph with color $g$.

Theorem 3. Assume that $\left(G^{r}, G^{b}, G^{g}\right)$ is a 3-edge coloring of $K_{17,17}$, where $K_{2,2} \nsubseteq G^{r}, K_{2,2} \nsubseteq G^{b}$, and $K_{3,3} \nsubseteq G^{g}$. So:

(a) $\left|E\left(G^{g}\right)\right|=141$.

(b) $\Delta\left(G^{g}\right)=9$ and $\delta\left(G^{g}\right)=8$.

(c) $D_{G^{g}}(X)=D_{G^{g}}(Y)=(9,9,9,9,9,8,8, \ldots, 8)$.

Proof of Theorem 3. Assume that $X=\left\{x_{1}, x_{2}, \ldots, x_{17}\right\}, Y=\left\{y_{1}, y_{2}, \ldots, y_{17}\right\}$ is a partition set of $K=K_{17,17}$ and $\left(G^{r}, G^{b}, G^{g}\right)$ is a 3-edge coloring of $K$, where $K_{2,2} \nsubseteq G^{r}, K_{2,2} \nsubseteq G^{b}$, and $K_{3,3} \nsubseteq G^{g}$. Since $|E(K)|=289$, if $\left|E\left(G^{g}\right)\right| \leq 140$ then $\left|E\left(\overline{G^{g}}\right)\right| \geq 149$-that is, either $\left|E\left(G^{r}\right)\right| \geq 75$ or $\left|E\left(G^{b}\right)\right| \geq 75$. In any case, by Proposition 1 , either $K_{2,2} \subseteq G^{r}$ or $K_{2,2} \subseteq G^{b}$, a contradiction. Hence, assume that $\left|E\left(G^{g}\right)\right| \geq 141$. If $\left|E\left(G^{g}\right)\right| \geq 142$ then by Proposition 1 , $K_{3,3} \subseteq G^{g}$, a contradiction again; that is, $\left|E\left(G^{g}\right)\right|=141$ and part $(a)$ is true.

To prove part $(b)$, since $\left|E\left(G^{g}\right)\right|=141$ by part $(a)$, we can check that $\Delta\left(G^{g}\right) \geq 9$. Assume that there exists a vertex of $V(K)$ say $x$, such that $\left|N_{G^{g}}(x)\right| \geq 10$ - that is, $\Delta\left(G^{g}\right) \geq$ 10. Consider $x$ and set $G_{1}^{g}=G^{g} \backslash\{x\}$, hence by part $(a),\left|E\left(G_{1}^{g}\right)\right| \leq 141-10=131$. Therefore, since $\left|E\left(K_{16,17}\right)\right|=272$, so $\left|E\left(\overline{G_{1}^{g}}\right)\right| \geq 141$-that is, either $\left|E\left(G_{1}^{r}\right)\right| \geq 71$ or $\left|E\left(G_{1}^{b}\right)\right| \geq 71$. In any case, by Proposition 1 either $K_{2,2} \subseteq G_{1}^{r} \subseteq G^{r}$ or $K_{2,2} \subseteq G_{1}^{b} \subseteq G^{b}$, a contradiction. So, $\Delta\left(G^{g}\right)=9$. To prove $\delta\left(G^{g}\right)=8$, assume that $M=\left\{x \in X,\left|N_{G^{g}}(x)\right|=\right.$ 9\} and $N=\left\{x \in X,\left|N_{G^{g}}(x)\right|=8\right\}$; by part (a) one can say that $|M| \geq 5$, if $|M|=6$, then $\delta\left(G^{g}\right) \leq 7$-that is, there is a vertex of $X$ (say $x$ ) such that $\left|N_{G^{g}}(x)\right| \leq 7$; therefore, 
$|N| \leq 10$. If $|N|=10$, then $\left|E\left(G^{8}[M \cup N, Y]\right)\right|=134$, so by Proposition $1, K_{3,3} \subseteq G^{8}$, a contradiction. Now assume that $|N| \leq 9$, thus $\left|E\left(G^{8}\right)\right| \leq(6 \times 9)+(9 \times 8)+(2 \times 7)=140$, a contradiction again. For $|M|=7$ if $|N| \geq 6$, then $\left|E\left(G^{g}\left[M \cup N^{\prime}, Y\right]\right)\right|=111$, where $N^{\prime} \subseteq N$ and $\left|N^{\prime}\right|=6$, so by Proposition $1, K_{3,3} \subseteq G^{8}$, a contradiction. Hence assume that $|N| \leq 5$; therefore, $\left|E\left(G^{8}\right)\right| \leq(7 \times 9)+(5 \times 8)+(5 \times 7)=138$, a contradiction again For $|M|=8$ if $|N| \geq 5$, then $\left|E\left(G^{g}\left[M \cup N^{\prime}, Y\right]\right)\right|=112$, where $N^{\prime} \subseteq N$ and $\left|N^{\prime}\right|=5$; therefore, by Proposition $1, K_{3,3} \subseteq G^{8}$, a contradiction, so assume that $|N| \leq 4$-that is, $\left|E\left(G^{g}\right)\right| \leq(8 \times 9)+(4 \times 8)+(5 \times 7)=139$, a contradiction again. For $|M|=9$ if $|N| \geq 3$, then $\left|E\left(G^{8}\left[M \cup N^{\prime}, Y\right]\right)\right|=105$, where $N^{\prime} \subseteq N$ and $\left|N^{\prime}\right|=3$, so by Proposition $1, K_{3,3} \subseteq G^{g}$, a contradiction. Thus $|N| \leq 2$-that is, $\left|E\left(G^{g}\right)\right| \leq(9 \times 9)+(2 \times 8)+(6 \times 7)=139$, which is a contradiction again. For $|M|=10$, if $|N| \geq 1$, then $\left|E\left(G^{g}\left[M \cup N^{\prime}, Y\right]\right)\right|=98$, where $N^{\prime} \subseteq N$ and $\left|N^{\prime}\right|=1$; so, by Proposition $1 K_{3,3} \subseteq G^{g}$, a contradiction. Thus, assume that $|N|=0$, so $\left|E\left(G^{g}\right)\right| \leq(10 \times 9)+(7 \times 7)=139$, a contradiction again. Therefore, $|M|=5$ and $|N|=12$-that is, $\delta\left(G^{g}\right)=8$, and part $(b)$ is true.

Now, by parts $(a)$ and $(b)$ it is straightforward to say that $D_{\mathrm{G}^{g}}(X)=D_{\mathrm{G}^{g}}(Y)=$ $(9,9,9,9,9,8,8, \ldots, 8)$ - that is, part $(c)$ is true, and this completes the proof.

\section{Proof of the Main Theorem}

In this section, by using the results of Section 2, we will prove the main theorem.

Suppose that $\left(G^{r}, G^{b}, G^{g}\right)$ is a 3-edge coloring of $K_{17,17}$, where $K_{2,2} \nsubseteq G^{r}, K_{2,2} \nsubseteq G^{b}$ and $K_{3,3} \nsubseteq G^{g}$. In the following theorem, we discuss the maximum number of common neighbors of $G^{g}(x)$ and $G^{g}\left(x^{\prime}\right)$ for $x, x^{\prime} \in X$.

Theorem 4. Assume that $\left(G^{r}, G^{b}, G^{g}\right)$ is a 3-edge coloring of $K_{17,17}$, where $K_{2,2} \nsubseteq G^{r}, K_{2,2} \nsubseteq G^{b}$ and $K_{3,3} \nsubseteq G^{g}$. Let $\left|N_{G^{g}}(x)\right|=9$ and $N_{G^{g}}(x)=Y_{1}$; the following results are true:

(a) For each $x \in X \backslash\left\{x_{1}\right\}$, we have $\left|N_{G^{g}}(x) \cap Y_{1}\right| \leq 5$.

(b) Assume that $n=\sum_{i=1}^{i=17}\left|N_{G^{g}}\left(x_{i}\right) \cap Y_{1}\right|$, then $72 \leq n \leq 73$.

Proof of Theorem 4. Assume that $X=\left\{x_{1}, x_{2}, \ldots, x_{17}\right\}, Y=\left\{y_{1}, y_{2}, \ldots, y_{17}\right\}$ is a partition set of $K=K_{17,17}$, and $\left(G^{r}, G^{b}, G^{g}\right)$ is a 3-edge coloring of $K$, where $K_{2,2} \nsubseteq G^{r}, K_{2,2} \nsubseteq G^{b}$ and $K_{3,3} \not \subseteq G^{8}$. Without loss of generality (W .l.g.) assume that $x=x_{1}$ and $Y_{1}=\left\{y_{1}, \ldots, y_{9}\right\}$. To prove part (a), by contrast assume that there exists a vertex of $X \backslash\left\{x_{1}\right\}$ (say $x$ ) such that $\left|N_{G^{g}}(x) \cap Y_{1}\right| \geq 6$. W.l.g., suppose that $x=x_{2}$ and $Y_{2}=\left\{y_{1}, y_{2}, \ldots, y_{6}\right\} \subseteq N_{G^{g}}\left(x_{2}\right)$. Since $K_{3,3} \not \subseteq G^{g}$, for each $x \in X \backslash\left\{x_{1}, x_{2}\right\}$, so $\left|N_{G^{g}}(x) \cap Y_{2}\right| \leq 2$-that is, $\sum_{i=1}^{i=17}\left|N_{G^{g}}\left(x_{i}\right) \cap Y_{2}\right| \leq$ $6+6+(15 \times 2) \leq 42$. Now, since $\left|E\left(G^{8}\left[X, Y_{2}\right]\right)\right| \leq 42$, one can check that there exists at least one vertex of $Y_{2}$ (say $y$ ), such that $\left|N_{G^{g}}(y)\right| \leq 7$, a contradiction to part $(c)$ of Theorem 3. Hence, $\left|N_{\mathrm{G}^{g}}(x) \cap Y_{1}\right| \leq 5$ for each $x \in X \backslash\left\{x_{1}\right\}$ 一that is, part $(a)$ is true.

To prove part (b), if $n \leq 71$, then by part (c) of Theorem 3, it can be checked that there exists at least one vertex of $Y_{1}$ (say $y$ ), such that $\left|N_{G^{8}}(y)\right| \leq 7$, a contradiction. Therefore, $n \geq 72$. Assume that $n \geq 74$ and let $D_{G^{g}}\left(Y_{1}\right)=\left(d_{1}, d_{2}, \ldots, d_{9}\right)$. Since $\sum_{i=1}^{i=17}\left|N_{G^{g}}\left(x_{i}\right) \cap Y_{1}\right| \geq$ 74 , there exist at least two vertices of $Y_{1}\left(\right.$ say $\left.y^{\prime}, y^{\prime \prime}\right)$, such that $\left|N_{G^{g}}\left(y^{\prime}\right)\right|=\left|N_{G^{g}}\left(y^{\prime \prime}\right)\right|=9$. Since $n \geq 74$ and $\left|X \backslash\left\{x_{1}\right\}\right|=16$, there exists at least one vertex of $X \backslash\left\{x_{1}\right\}$ (say $x^{\prime}$ ), such that $\left|N_{G^{g}}\left(x^{\prime}\right) \cap Y_{1}\right|=5$. W.l.g., suppose that $x^{\prime}=x_{2}$ and $N_{G^{g}}\left(x_{2}\right) \cap Y_{1}=Y_{2}=\left\{y_{1}, \ldots, y_{5}\right\}$. Now we have the following claims:

Claim 1. For each $x \in X \backslash\left\{x_{1}, x_{2}\right\}$, we have $\left|N_{G^{g}}(x) \cap Y_{2}\right|=2$ and $D_{G^{g}}\left(Y_{2}\right)=(8,8,8,8,8)$.

Proof of Claim 1. Since $K_{3,3} \nsubseteq G^{g}$ for each $x \in X \backslash\left\{x_{1}, x_{2}\right\}$, thus $\left|N_{G^{g}}(x) \cap Y_{2}\right| \leq 2$-that is, $\sum_{i=1}^{i=17}\left|N_{G^{g}}\left(x_{i}\right) \cap Y_{2}\right| \leq 5+5+(15 \times 2) \leq 40$. Now, since $\left|E\left(G^{g}\left[X, Y_{2}\right]\right)\right| \leq 40$ and $\left|Y_{2}\right|=5$, if there exists a vertex of $\left.X_{1}\right)\left(\right.$ say $\left.x^{\prime}\right)$, such that $\left|N_{G^{g}}(x) \cap Y_{2}\right| \leq 1$, then $\left|E\left(G^{g}\left[X, Y_{2}\right]\right)\right| \leq$ 
39; therefore, there exists at least one vertex of $Y_{2}$ (say $y$ ), such that $\left|N_{G^{g}}(y)\right| \leq 7$, a contradiction to part $(c)$ of Theorem 3. So, $\left|N_{G^{g}}(x) \cap Y_{2}\right|=2$ and $\sum_{y \in Y_{2}}\left|N_{G^{g}}(y)\right|=40$, therefore by part $(c)$ of Theorem $3 D_{G^{g}}\left(Y_{2}\right)=(8,8,8,8,8)$, and the proof of the claim is complete.

Claim 2. $D_{G^{g}}\left(X_{1}\right)=(5,4,4, \ldots, 4)$ where $X_{1}=X \backslash\left\{x_{1}\right\}$, in other word $\left|N_{G^{g}}\left(x_{i}\right) \cap Y_{1}\right|=4$ for each $i \in\{3,4, \ldots, 17\}$.

Proof of Claim 2. By contradiction, assume that there exists a vertex of $X \backslash\left\{x_{1}, x_{2}\right\}$ (say $x$ ), such that $\left|N_{G g}(x) \cap Y_{1}\right|=5$. W.1.g suppose that $x=x_{3}$ and $N_{G^{g}}\left(x_{3}\right) \cap Y_{1}=Y_{3}$, now by Claim 1, $\left|N_{G^{g}}\left(x_{3}\right) \cap Y_{2}\right|=2$. W.l.g., assume that $Y_{3}=\left\{y_{1}, y_{2}, y_{6}, y_{7}, y_{8}\right\}$, thus by Claim 1, $D_{G^{g}}\left(Y_{3}\right)=(8,8,8,8,8)$-that is, $\left|N_{G^{g}}(y)\right|=8$ for each $y \in Y_{1} \backslash\left\{y_{9}\right\}$. Since $\Delta\left(G^{g}\right)=9$, we can check that $n=\sum_{i=1}^{i=17}\left|N_{G^{g}}\left(x_{i}\right) \cap Y_{1}\right|=\sum_{i=1}^{i=9}\left|N_{G^{g}}\left(y_{i}\right)\right| \leq(8 \times 8)+9=73$, a contradiction. So, $D_{G^{g}}\left(X_{1}\right)=(5,4,4, \ldots, 4)$, and the proof of the claim is complete.

Assume that $N_{G^{g}}\left(x_{2}\right) \cap Y_{1}=Y_{2}=\left\{y_{1} \ldots, y_{5}\right\}$, by Claim $1 D_{G^{g}}\left(Y_{2}\right)=(8,8,8,8,8)$. Since there exist at lest two vertices of $Y_{1}$ (say $\left.y^{\prime}, y^{\prime \prime}\right)$, such that $\left|N_{G^{g}}\left(y^{\prime}\right)\right|=\left|N_{G^{g}}\left(y^{\prime \prime}\right)\right|=9$, thus $y^{\prime}, y^{\prime \prime} \in\left\{y_{6}, y_{7}, y_{8}, y_{9}\right\}$. W.l.g., we can suppose that $y^{\prime}=y_{6}$ and $N_{G^{g}}\left(y_{6}\right)=X_{2}=$ $\left\{x_{1}, x_{3}, \ldots, x_{10}\right\}$. By Claim 2, $\left|N_{G^{g}}(x) \cap Y_{1}\right|=4$ and $\left|N_{G^{g}}(x) \cap Y_{2}\right|=2$ for each $x \in$ $X_{2} \backslash\left\{x_{1}\right\}$-that is, $\left|N_{G^{g}}(x) \cap\left\{y_{7}, y_{8}, y_{9}\right\}\right|=1$ for each $x \in X_{2} \backslash\left\{x_{1}\right\}$. Since $\left|X_{2} \backslash\left\{x_{1}\right\}\right|=8$ and $\left|N_{G^{8}}(x) \cap\left\{y_{7}, y_{8}, y_{9}\right\}\right|=1$, by the pigeon-hole principle, there exists a vertex of $\left\{y_{7}, y_{8}, y_{9}\right\}$ (say $y$ ), such that $\left|N_{G g}(y) \cap X_{2} \backslash\left\{x_{1}\right\}\right| \geq 3$. W.l.g., we can suppose that $y=y_{7}$ and $\left\{x_{3}, x_{4}, x_{5}\right\} \subseteq N_{G^{g}}\left(y_{7}\right) \cap X_{2} \backslash\left\{x_{1}\right\}$. As $\left|Y_{2}\right|=5$ and $\left|N_{G^{g}}\left(x_{i}\right) \cap Y_{2}\right|=2$ for $i=3,4,5$, there exist $i, i^{\prime} \in\{3,4,5\}$, such that $\left|N_{G^{g}}\left(x_{i}\right) \cap N_{G^{g}}\left(x_{i^{\prime}}\right) \cap Y_{2}\right| \neq 0$. W.1.g., suppose that $i=$ $3, i^{\prime}=4$ and $y_{1} \in N_{G^{g}}\left(x_{3}\right) \cap N_{G^{g}}\left(x_{4}\right) \cap Y_{2}$. Therefore, $K_{3,3} \subseteq G^{g}\left[\left\{x_{1}, x_{3}, x_{4}\right\},\left\{y_{1}, y_{6}, y_{7}\right\}\right]$, a contradiction. So, $n \leq 73$ and the proof of the theorem is complete.

In part (b) of Theorem 4, we showed that $72 \leq n=\sum_{i=1}^{i=17}\left|N_{G^{g}}\left(x_{i}\right) \cap Y_{1}\right| \leq 73$. Now we consider these two cases independently.

\subsection{The Case That $n=73$}

In the following theorem, we prove that in any 3-edge coloring of $K_{17,17}$ (say $\left(G^{r}, G^{b}, G^{g}\right)$, where $K_{2,2} \nsubseteq G^{r}, K_{2,2} \nsubseteq G^{b}$ ), if there exists a vertex of $V(K)$ (say $x$ ), such that $\left|N_{G^{g}}(x)\right|=9$ and $\sum_{x_{i} \in X \backslash\{x\}}\left|N_{G^{g}}\left(x_{i}\right) \cap N_{G^{g}}(x)\right|=64$, then $K_{3,3} \subseteq G^{g}$.

Theorem 5. Assume that $\left(G^{r}, G^{b}, G^{g}\right)$ is a 3-edge coloring of $K=K_{17,17}$, such that $K_{2,2} \nsubseteq G^{r}$, $K_{2,2} \nsubseteq G^{b}$. Assume that there exists a vertex of $V(K)$ (say $\left.x\right)$, such that $\left|N_{G^{g}}(x)\right|=9$. If $\sum_{i=1}^{i=17}\left|N_{G^{g}}\left(x_{i}\right) \cap Y_{1}\right|=73$ where $Y_{1}=N_{G^{g}}(x)$, then $K_{3,3} \subseteq G^{g}$.

Proof of Theorem 5. By contradiction, assume that $K_{3,3} \nsubseteq G^{g}$. Therefore, by Theorem 3 and Theorem 4, we have the following results:

(a) $\left|E\left(G^{g}\right)\right|=141$.

(b) $\Delta\left(G^{g}\right)=9$ and $\delta\left(G^{g}\right)=8$.

(c) $D_{G^{g}}(X)=D_{G^{g}}(Y)=(9,9,9,9,9,8,8, \ldots, 8)$.

(d) For each $x^{\prime} \in X \backslash\{x\}$ we have $\left|N_{G^{g}}(x) \cap N_{G^{g}}\left(x^{\prime}\right)\right| \leq 5$.

(e) If $A=\left\{x \in X,\left|N_{G^{g}}(x)\right|=9\right\}$, then $|A|=5$ and $72 \leq \sum_{y \in N_{G^{g}}(x)}\left|N_{G^{g}}(y)\right| \leq 73$, for each $x \in A$.

Assume that $X=\left\{x_{1}, x_{2}, \ldots, x_{17}\right\}, Y=\left\{y_{1}, y_{2}, \ldots, y_{17}\right\}$ is the partition set of $K=$ $K_{17,17}$, and $\left(G^{r}, G^{b}, G^{g}\right)$ is a 3-edge coloring of $K$, where $K_{2,2} \nsubseteq G^{r}, K_{2,2} \nsubseteq G^{b}$ and $K_{3,3} \nsubseteq G^{g}$. 
W.l.g., assume that $x=x_{1}, Y_{1}=\left\{y_{1}, y_{2}, \ldots, y_{9}\right\}$, and $n=\sum_{i=1}^{i=17}\left|N_{G^{g}}\left(x_{i}\right) \cap Y_{1}\right|=73$. Since $n=73$, by $(c)$ we can say that $D_{G^{8}}\left(Y_{1}\right)=\left(d_{1}, d_{2}, \ldots, d_{9}\right)=(9,8,8, \ldots, 8)$ - that is, there exists a vertex of $Y_{1}$ (say $y$ ), such that $\left|N_{G 8}(y)\right|=9$. By (d), $\left|N_{G^{g}}\left(x_{1}\right) \cap N_{G^{g}}(x)\right| \leq 5$ for each $x \in X\left\{\backslash x_{1}\right\}$. Set $C=\left\{x \in X,\left|N_{G^{g}}(x) \cap N_{G^{g}}\left(x_{1}\right)\right|=5\right\}$. Now by argument similar to the proof of Claim 1, we have the following claim:

Claim 3. Assume that $x \in C$ and $N_{G^{g}}(x) \cap Y_{1}=Y^{\prime}$, then for each $x^{\prime} \in X \backslash\left\{x_{1}, x\right\}$, we have $\left|N_{G^{g}}\left(x^{\prime}\right) \cap Y^{\prime}\right|=2$ and $D_{G^{g}}\left(Y^{\prime}\right)=(8,8,8,8,8)$.

Here there exists a claim about $|C|$ as follows:

Claim 4. $|C| \leq 2$.

Proof of Claim 4. By contradiction, assume that $|C| \geq 3$. W.l.g., suppose that $\left\{x_{2}, x_{3}, x_{4}\right\} \subseteq$ $C$ and $N_{G^{g}}\left(x_{2}\right) \cap Y_{1}=Y_{2}=\left\{y_{1}, \ldots, y_{5}\right\}$. By Claim 3, $\left|N_{G^{g}}(x) \cap Y_{2}\right|=2$ for each $x \in X \backslash\left\{x_{1}, x_{2}\right\}$. W.l.g., suppose that $N_{G^{g}}\left(x_{3}\right) \cap Y_{1}=Y_{3}=\left\{y_{1}, y_{2}, y_{6}, y_{7}, y_{8}\right\}$. Since $x_{4} \in C$ and $\left|N_{G^{g}}\left(x_{4}\right) \cap Y_{i}\right|=2$ for $i=2,3, y_{9} \in N_{G^{g}}\left(x_{4}\right) \cap Y_{1}$. Hence, for each $y \in Y_{1}$, there is at least one $i \in\{2,3,4\}$ such that $y \in N_{G^{g}}\left(x_{i}\right)$; therefore, by Claim $3, D_{G^{g}}\left(Y_{1}\right)=$ $(8,8,8,8,8,8,8,8,8)$, which is in contrast to $\sum_{i=1}^{i=17}\left|N_{G^{g}}\left(x_{i}\right) \cap Y_{1}\right|=\sum_{i=1}^{i=9}\left|N_{G^{g}}\left(y_{i}\right)\right|=73$, so $|C| \leq 2$.

Now by considering $|C|$ there are three cases as follows:

Case 1: $|C|=0$. Since $n=73,\left|Y_{1}\right|=9$ and $|C|=0, D_{G^{g}}\left(X \backslash\left\{x_{1}\right\}\right)=(4,4, \ldots, 4,4)$, $D_{G^{g}}\left(Y_{1}\right)=(9,8,8,8,8,8,8,8,8), \sum_{i=1}^{i=17}\left|N_{G^{g}}\left(x_{i}\right) \cap Y^{\prime}\right|=68$, and $D_{G^{g}}\left(Y^{\prime}\right)=(9,9,9,9,8,8,8,8)$, where $Y^{\prime}=Y \backslash Y_{1}$. Set $B=\left\{y \in Y^{\prime},\left|N_{G^{g}}(y)\right|=9\right\}$, so $|B|=4$.

Now we are ready to prove the following claim:

Claim 5. There exists a vertex of $A \backslash\left\{x_{1}\right\}$ (say $x$ ), such that:

$$
\sum_{y \in N_{G^{g}}(x)}\left|N_{G^{g}}(y)\right| \geq 74,
$$

in which $A=\left\{x \in X,\left|N_{G^{g}}(x)\right|=9\right\}$.

Proof of Claim 5. $D_{G^{g}}\left(X_{1}\right)=(4,4, \ldots, 4,4)$ and $D_{G^{g}}\left(Y_{1}\right)=(9,8,8,8,8,8,8,8,8)$ for each $x \in A \backslash\left\{x_{1}\right\}$; thus:

$$
\sum_{y \in N_{G^{g}}(x) \cap Y_{1}}\left|N_{G^{g}}(y)\right| \geq 32
$$

As $\left|Y^{\prime}\right|=8,|B|=4$, and $\left|N_{G^{g}}\left(x_{i}\right) \cap Y^{\prime}\right|=5$ for each $x \in A \backslash\left\{x_{1}\right\}$, there exists at least one vertex of $A \backslash\left\{x_{1}\right\}$ (say $x$ ), such that $\left|N_{G^{g}}(x) \cap B\right| \geq 2$, otherwise $K_{3,3} \subseteq G^{g}\left[A, Y^{\prime} \backslash B\right]$, a contradiction. Hence, w.l.g., suppose that $x_{2} \in A$, where $\left|N_{G^{g}}\left(x_{2}\right) \cap B\right| \geq 2$. So:

$$
\sum_{y \in N_{G^{g}}\left(x_{2}\right) \cap \gamma^{\prime}}\left|N_{G^{g}}(y)\right| \geq 42 .
$$

That is,

$$
\sum_{y \in N_{G^{g}}\left(x_{2}\right)}\left|N_{G^{g}}(y)\right|=\sum_{y \in N_{G^{g}}\left(x_{2}\right) \cap Y^{\prime}}\left|N_{G^{g}}(y)\right|+\sum_{y \in N_{G^{g}}(x) \cap Y_{1}}\left|N_{G^{g}}(y)\right| \geq 42+32=74 .
$$

Now by considering $x_{2}$ and $N_{G^{g}}\left(x_{2}\right)$ and by (e) (or part (b) of Theorem 4) $K_{3,3} \subseteq G^{g}$, a contradiction again. 
Case 2: $|C|=1$. W.l.g., suppose that $C=\left\{x_{2}\right\}, N_{G^{g}}\left(x_{2}\right) \cap Y_{1}=Y_{2}=\left\{y_{1}, \ldots, y_{5}\right\}$. By Claim $3,\left|N_{G^{8}}\left(x_{2}\right) \cap N_{G^{8}}(x) \cap Y_{1}\right|=2$ for each $x \in X \backslash\left\{x_{1}, x_{2}\right\}$ and $\left|N_{G^{8}}\left(y_{i}\right)\right|=8$ for each $i \in\{1,2, \ldots, 5\}$. Since there exists a vertex of $Y_{1}$ named $y$, such that $\left|N_{G^{g}}(y)\right|=9$, w.l.g. we can suppose that $y=y_{6}$ and $N_{G^{g}}\left(y_{6}\right)=\left\{x_{1}, x_{3}, x_{4} \ldots, x_{10}\right\}$. Since $n=73$ and $|C|=1, D_{G^{g}}\left(X_{1}\right)=(5,4,4, \ldots, 4,3)$-that is, there exist at least seven vertices of $N_{G^{g}}\left(y_{6}\right) \backslash\left\{x_{1}\right\}$ (say $X_{3}=\left\{x_{3}, x_{4} \ldots, x_{9}\right\}$ ), such that $\left|N_{G^{g}}(x) \cap Y_{1}\right|=4$ for each $x \in X_{3}$. Since $\left|X_{3}\right|=7,\left|Y_{2}\right|=5,\left|N_{G^{g}}(x) \cap Y_{1}\right|=4$ and $\left|N_{G^{g}}\left(x_{i}\right) \cap Y_{2}\right|=2$ for each $x \in X_{3}$, $\left|N_{G^{g}}(x) \cap\left\{y_{7}, y_{8}, y_{9}\right\}\right|=1$ for each $x \in X_{3}$. Therefore, by the pigeon-hole principle there exists a vertex of $\left\{y_{7}, y_{8}, y_{9}\right\}$ (say $\left.y^{\prime}\right)$, such that $\left|N_{G^{8}}\left(y^{\prime}\right) \cap X_{3}\right| \geq 3$. W.1.g., suppose that $y^{\prime}=y_{7}$ and $\left\{x_{3}, x_{4}, x_{5}\right\} \subseteq N_{G^{8}}\left(y_{7}\right)$. Therefore, since $\left|Y_{2}\right|=5$, there exists $i, i^{\prime} \in$ $\{3,4,5\}$ such that $\left|N_{G^{8}}\left(x_{i}\right) \cap N_{G^{g}}\left(x_{i^{\prime}}\right) \cap Y_{2}\right| \neq 0$. W.1.g., suppose that $i=3, i^{\prime}=4$ and $y_{1} \in N_{G^{8}}\left(x_{3}\right) \cap N_{G^{8}}\left(x_{4}\right) \cap Y_{2}$. Therefore, $K_{3,3} \subseteq G^{g}\left[\left\{x_{1}, x_{3}, x_{4}\right\},\left\{y_{1}, y_{6}, y_{7}\right\}\right]$, which is a contradiction.

Case 3: $|C|=2$. W.l.g., suppose that $C=\left\{x_{2}, x_{3}\right\}, N_{G^{g}}\left(x_{2}\right) \cap Y_{1}=Y_{2}=\left\{y_{1}, \ldots, y_{5}\right\}$. By Claim 3, $\left|N_{G^{g}}\left(x_{2}\right) \cap N_{G^{g}}\left(x_{3}\right) \cap Y_{1}\right|=2$. So, w.l.g. we can suppose that $N_{G^{g}}\left(x_{3}\right) \cap$ $Y_{1}=Y_{3}=\left\{y_{1}, y_{2}, y_{6}, y_{7}, y_{8}\right\}$. Now, by Claim $3,\left|N_{G^{8}}\left(y_{i}\right)\right|=8$ for each $i \in\{1,2, \ldots, 8\}$. Since there is a vertex of $Y_{1}$ named $y$, such that $\left|N_{G^{g}}(y)\right|=9, y=y_{9}$. W.l.g., we can assume that $N_{G^{8}}\left(y_{9}\right)=X_{2}=\left\{x_{1}, x_{4}, x_{5} \ldots, x_{11}\right\}$. Since $n=73$ and $|C|=2, D_{G^{8}}\left(X_{1}\right)=$ $(5,5,4,4, \ldots, 4,3,3)$ - that is, there exist two vertices of $X$ (say $\left.x, x^{\prime}\right)$, such that $\mid N_{G^{g}}(x) \cap$ $Y_{1} \mid=3$. If $\left|N_{G^{g}}\left(y_{9}\right) \cap\left\{x, x^{\prime}\right\}\right| \leq 1$, then there exist at least seven vertices of $N_{G^{g}}\left(y_{9}\right) \backslash\left\{x_{1}\right\}$, such that $\left|N_{G^{g}}(x) \cap Y_{1}\right|=4$; in this case, the proof is the same as Case 1. Hence, assume that $x, x^{\prime} \in N_{G^{g}}\left(y_{9}\right)$. Since $\left|N_{G^{g}}(x) \cap Y_{2}\right|=\left|N_{G^{g}}\left(x^{\prime}\right) \cap Y_{2}\right|=2$, one can check that $\left|N_{G^{g}}(x) \cap\left\{y_{6}, y_{7}, y_{8}\right\}\right|=\left|N_{G^{g}}\left(x^{\prime}\right) \cap\left\{y_{6}, y_{7}, y_{8}\right\}\right|=0$. Assume that $X_{i}=N_{G^{g}}\left(y_{i}\right)$ for $i=$ $6,7,8$. Since $\left|X_{i}\right|=8$ and $x, x^{\prime} \notin X_{i}$, then for each $x \in X_{i} \backslash\left\{x_{1}\right\}$ we have $\left|N_{G^{g}}(x) \cap Y_{1}\right|=4$. Therefore, by considering $X_{i} \backslash\left\{x_{1}\right\}$ and $y_{i}$ for each $i \in\{6,7,8\}$, the proof is the same as Case 1 and $K_{3,3} \subseteq G^{g}$, a contradiction again.

Therefore, by Cases 1, 2, and 3 the assumption does not hold-that is, $K_{3,3} \subseteq G^{g}$ and this completes the proof of the theorem.

\subsection{The Case That $n=72$}

In the following theorem, we prove that in any 3-edge coloring of $K_{17,17}$ (say $\left(G^{r}, G^{b}, G^{g}\right)$, where $K_{2,2} \nsubseteq G^{r}, K_{2,2} \nsubseteq G^{b}$ ), if there exists a vertex of $V(K)$ (say $x$ ), such that $\left|N_{G^{g}}(x)\right|=9$ and $\sum_{x_{i} \in X \backslash\{x\}}\left|N_{G^{g}}\left(x_{i}\right) \cap N_{G^{g}}(x)\right|=63$, then $K_{3,3} \subseteq G^{g}$.

Theorem 6. Assume that $\left(G^{r}, G^{b}, G^{g}\right)$ is a 3-edge coloring of $K=K_{17,17}$, where $K_{2,2} \nsubseteq G^{r}$, $K_{2,2} \nsubseteq G^{b}$. Suppose that there exists a vertex of $V(K)$ (say $\left.x\right)$, such that $\left|N_{G^{g}}(x)\right|=9$. If $\sum_{i=1}^{i=17}\left|N_{G^{g}}\left(x_{i}\right) \cap Y_{1}\right|=72$, where $Y_{1}=N_{G^{g}}(x)$, then $K_{3,3} \subseteq G^{g}$.

Proof of Theorem 6 . By contradiction, assume that $K_{3,3} \nsubseteq G^{g}$. Therefore, by Theorems 3 and 4, we have the following results:

(a) $\left|E\left(G^{g}\right)\right|=141$.

(b) $\Delta\left(G^{g}\right)=9$ and $\delta\left(G^{g}\right)=8$.

(c) $D_{G^{g}}(X)=D_{G^{g}}(Y)=(9,9,9,9,9,8,8, \ldots, 8)$.

(d) For each $x \in X \backslash\left\{x_{1}\right\}$, we have $\left|N_{G^{g}}(x) \cap Y_{1}\right| \leq 5$.

(e) If $A=\left\{x \in X,\left|N_{G^{g}}(x)\right|=9\right\}$, then $|A|=5$ and $72 \leq \sum_{y \in N_{G^{g}}(x)}\left|N_{G^{g}}(y)\right| \leq 73$, for each $x \in A$.

Assume that $X=\left\{x_{1}, x_{2}, \ldots, x_{17}\right\}, Y=\left\{y_{1}, y_{2}, \ldots, y_{17}\right\}$ is a partition set of $K=K_{17,17}$, and $\left(G^{r}, G^{b}, G^{g}\right)$ is a 3-edge coloring of $K$, where $K_{2,2} \nsubseteq G^{r}, K_{2,2} \nsubseteq G^{b}$ and $K_{3,3} \nsubseteq G^{g}$. W.l.g., assume that $x=x_{1}, Y_{1}=\left\{y_{1}, y_{2}, \ldots, y_{9}\right\}$, and $n=\sum_{i=1}^{i=17}\left|N_{G^{g}}\left(x_{i}\right) \cap Y_{1}\right|=72$. Since 
$n=73$, by $(c)$ we can say that $D_{G^{g}}\left(Y_{1}\right)=\left(d_{1}, d_{2}, \ldots, d_{9}\right)=(8,8,8, \ldots, 8)$. Set $C=\{x \in$ $\left.X,\left|N_{G^{g}}(x) \cap N_{G^{g}}\left(x_{1}\right)\right|=5\right\}$. Define $D$ and $E$ as follows:

$$
\begin{aligned}
& D=\left\{x \in X \backslash\left\{x_{1}\right\}, \text { such that }\left|N_{G^{g}}(x) \cap Y_{1}\right|=5\right\} \\
& E=\left\{x \in X \backslash\left\{x_{1}\right\}, \text { such that }\left|N_{G^{g}}(x) \cap Y_{1}\right|=3\right\} .
\end{aligned}
$$

Here we have a claim about $|D|$ and $|E|$ as follows:

Claim 6. $|D| \leq 3$ and $|E| \leq 4$.

Proof of Claim 6. By contradiction, suppose that $|D| \geq 4$. W.l.g., assume that $\left\{x_{2}, x_{3}, x_{4}, x_{5}\right\} \subseteq$ $D, N_{G^{g}}\left(x_{2}\right) \cap Y_{1}=Y_{2}=\left\{y_{1}, \ldots, y_{5}\right\}$. Now, by Claim 3, $\left|N_{G^{g}}(x) \cap Y_{2}\right|=2$ for each $x \in X \backslash\left\{x_{1}, x_{2}\right\}$. W.l.g., we can suppose that $N_{G^{8}}\left(x_{3}\right) \cap Y_{1}=Y_{3}=\left\{y_{1}, y_{2}, y_{6}, y_{7}, y_{8}\right\}$. Consider $N_{G^{g}}\left(x_{i}\right) \cap Y_{1}(i=4,5)$. Since $\left|N_{G^{g}}\left(x_{i}\right) \cap Y_{j}\right|=2(i=4,5, j=2,3)$ and $x_{i} \in A,\left|N_{G^{8}}\left(x_{i}\right) \cap\left\{y_{3}, y_{4}, y_{5}\right\}\right|=2,\left|N_{G^{8}}\left(x_{i}\right) \cap\left\{y_{6}, y_{7}, y_{8}\right\}\right|=2$, and $y_{9} \in N_{G^{g}}\left(x_{i}\right)$ for $i=4,5$; otherwise, if there exists a vertex of $\left\{x_{4}, x_{5}\right\}$ (say $x$ ), such that $\mid N_{G^{g}}\left(x_{i}\right) \cap$ $\left\{y_{1}, y_{2}\right\} \mid \neq 2$, then $K_{3,3} \subseteq G^{8}\left[\left\{x_{1}, x_{i}, x\right\}, Y_{1}\right]$ for some $i \in\{1,2\}$, a contradiction. Therefore, since $\left|\left\{y_{3}, y_{4}, y_{5}\right\}\right|=\left|\left\{y_{6}, y_{7}, y_{8}\right\}\right|=3$ and $x_{4}, x_{5} \in A$, by the pigeon-hole principle $\left|N_{G^{g}}\left(x_{4}\right) \cap N_{G^{8}}\left(x_{5}\right) \cap\left\{y_{3}, y_{4}, y_{5}\right\}\right| \geq 1$ and $\left|N_{G^{8}}\left(x_{4}\right) \cap N_{G^{8}}\left(x_{5}\right) \cap\left\{y_{6}, y_{7}, y_{8}\right\}\right| \geq 1$. W.l.g., we can suppose that $y_{3}, y_{6} \in N_{G^{8}}\left(x_{4}\right) \cap N_{G^{8}}\left(x_{5}\right)$, since $y_{9} \in N_{G^{8}}\left(x_{4}\right) \cap N_{G^{8}}\left(x_{5}\right)$, so $K_{3,3} \subseteq G^{8}\left[\left\{x_{1}, x_{4}, x_{5}\right\},\left\{y_{3}, y_{6}, y_{9}\right\}\right]$, a contradiction. Therefore, $|D| \leq 3$. Now, as $\sum_{i=2}^{i=17}\left|N_{G^{g}}\left(x_{i}\right) \cap Y_{1}\right|=63$ and $|D| \leq 3$, we can say that $|E| \leq 4$ and the proof of the claim is complete.

Now, by considering $|D|$, there are three cases as follows:

Case 1: $|D|=0$. Since $n=72$ and $|D|=0, D_{G^{g}}\left(X \backslash\left\{x_{1}\right\}\right)=(4,4, \ldots, 4,3), D_{G^{g}}\left(Y_{1}\right)=$ $(8,8,8,8,8,8,8,8,8), \sum_{i=1}^{i=17}\left|N_{G^{g}}\left(x_{i}\right) \cap Y^{\prime}\right|=69$ and $D_{G^{8}}\left(Y^{\prime}\right)=(9,9,9,9,9,8,8,8)$, where $Y^{\prime}=Y \backslash Y_{1}$. Set $B=\left\{y \in Y^{\prime},\left|N_{G^{g}}(y)\right|=9\right\}$, hence $|B|=5$.

Now, we have the following claim:

Claim 7. There exists a vertex of $A \backslash\left\{x_{1}\right\}$ (say $x$ ), such that:

$$
\sum_{y \in N_{G^{g}}(x)}\left|N_{G^{g}}(y)\right| \geq 75,
$$

in which $A=\left\{x \in X,\left|N_{G^{g}}(x)\right|=9\right\}$.

Proof of Claim 7. Since $D_{G^{g}}\left(X_{1}\right)=(4,4, \ldots, 4,3)$ and $D_{G^{g}}\left(Y_{1}\right)=(8,8,8,8,8,8,8,8,8)$, so for at least three vertices of $A \backslash\left\{x_{1}\right\}$,

$$
\sum_{y \in N_{G^{g}}(x) \cap Y_{1}}\left|N_{G^{g}}(y)\right| \geq 32 .
$$

Therefore, since $\left|N_{G^{g}}\left(x_{i}\right) \cap Y^{\prime}\right|=5$ for each $x \in A \backslash\left\{x_{1}\right\}$ and $D_{G^{g}}\left(Y^{\prime}\right)=(9,9,9$, $9,9,8,8,8)$, there exists at least one vertex of $A \backslash\left\{x_{1}\right\}$ (say $x$ ), such that $\left|N_{G^{8}}(x) \cap B\right| \geq 3$; otherwise, $K_{3,3} \subseteq G^{8}\left[A, Y^{\prime} \backslash B\right]$, a contradiction. Hence, w.l.g., suppose that $x_{2} \in A$ and $\left|N_{G^{g}}\left(x_{2}\right) \cap B\right| \geq 3$; therefore:

$$
\sum_{y \in N_{G^{g}}(x) \cap Y^{\prime}}\left|N_{G^{g}}(y)\right| \geq 3 \times 9+2 \times 8=43 .
$$

That is, we have:

$$
\sum_{y \in N_{G^{g}}\left(x_{2}\right)}\left|N_{G^{g}}(y)\right|=\sum_{y \in N_{G^{g}}\left(x_{2}\right) \cap Y^{\prime}}\left|N_{G^{g}}(y)\right|+\sum_{y \in N_{G^{g}}(x) \cap Y_{1}}\left|N_{G^{g}}(y)\right| \geq 43+32=75 .
$$


Now, by considering $x_{2}$ and $N_{G^{g}}\left(x_{2}\right)$ and by $(e)$ (or by part $(b)$ of Theorem 4 ), $K_{3,3} \subseteq$ $G^{g}$, a contradiction again.

Case 2: $|D|=1$ (for the case that $|D|=2$, the proof is same). W.l.g., assume that $D=\left\{x_{2}\right\}, N_{G^{g}}\left(x_{2}\right) \cap Y_{1}=Y_{2}=\left\{y_{1}, \ldots, y_{5}\right\}$. Since $n=72,|D|=1$ and $\left|N_{G^{g}}(x) \cap Y_{1}\right| \leq 5$, $|E|=2$. As $\left|N_{G^{g}}(x) \cap Y_{2}\right|=2$ for each $x \in X \backslash\left\{x_{1}, x_{2}\right\}$ and $|E|=2$, there exists a vertex of $\left\{y_{6}, y_{7}, y_{8}, y_{9}\right\}$ (say $y$ ), such that for each vertex of $N_{G^{g}}(y) \cap X \backslash\left\{x_{1}\right\}$ (say $x$ ), $\mid N_{G^{g}}(x) \cap$ $Y_{1} \mid=4$. W.l.g., we can suppose that $y=y_{6}, N_{G^{8}}\left(y_{6}\right) \cap X \backslash\left\{x_{1}\right\}=\left\{x_{3}, x_{4}, \ldots, x_{9}\right\}$. Since $\left|N_{G^{8}}\left(y_{6}\right) \cap X \backslash\left\{x_{1}\right\}\right|=7$ and $\left|N_{G^{8}}(x) \cap Y_{2}\right|=2$ for each $x \in N_{G^{8}}\left(y_{6}\right) \cap X \backslash\left\{x_{1}\right\}$, $\left|N_{G^{g}}(x) \cap\left\{y_{7}, y_{8}, y_{9}\right\}\right|=1$. Therefore, by the pigeon-hole principle there exists a vertex of $\left\{y_{7}, y_{8}, y_{9}\right\}$ (say $y^{\prime}$ ), such that $\left|N_{G^{g}}\left(y_{6}\right) \cap N_{G^{g}}\left(y^{\prime}\right) \cap X \backslash\left\{x_{1}\right\}\right| \geq 3$. W.l.g., suppose that $y^{\prime}=y_{7}$ and $\left\{x_{3}, x_{4}, x_{5}\right\} \subseteq N_{G^{8}}\left(y_{6}\right) \cap N_{G^{8}}\left(y_{7}\right) \cap X \backslash\left\{x_{1}\right\}$. Therefore, since $\left|Y_{2}\right|=5$ and $\left|N_{G^{g}}(x) \cap Y_{2}\right|=2$, there exist at least two vertices of $\left\{x_{3}, x_{4}, x_{5}\right\}$ (say $x^{\prime}, x^{\prime \prime}$ ), such that $\left|N_{G^{g}}\left(x^{\prime}\right) \cap N_{G^{g}}\left(x^{\prime \prime}\right) \cap Y_{2}\right| \neq 0$. W.l.g., suppose that $x^{\prime}=x_{3}, x^{\prime \prime}=x_{4}$ and $y_{1} \in$ $N_{G^{g}}\left(x_{3}\right) \cap N_{G^{g}}\left(x_{4}\right)$. Therefore, $K_{3,3} \subseteq G^{g}\left[\left\{x_{1}, x_{3}, x_{4}\right\},\left\{y_{1}, y_{6}, y_{7}\right\}\right]$, a contradiction.

Case 3: $|D|=3$. W.1.g., suppose that $D=\left\{x_{2}, x_{3}, x_{4}\right\}, N_{G^{g}}\left(x_{2}\right) \cap Y_{1}=Y_{2}=$ $\left\{y_{1}, \ldots, y_{5}\right\}$. By Claim 3, $\left|N_{G^{g}}\left(x_{2}\right) \cap N_{G^{g}}\left(x_{3}\right) \cap Y_{1}\right|=2$. W.l.g., we can assume that $N_{G^{g}}\left(x_{3}\right) \cap Y_{1}=Y_{3}=\left\{y_{1}, y_{2}, y_{6}, y_{7}, y_{8}\right\}$. Since $x_{4} \in D$ and $\left|N_{G^{g}}\left(x_{4}\right) \cap Y_{i}\right|=2$ for $i=2,3$, $y_{9} \in N_{G^{g}}\left(x_{4}\right)$. If $\left|N_{G^{g}}\left(x_{4}\right) \cap\left\{y_{1}, y_{2}\right\}\right| \neq 0$, as $\left|N_{G^{g}}\left(x_{2}\right) \cap N_{G^{g}}\left(x_{4}\right) \cap Y_{1}\right|=2$ and $x_{4} \in D$, one can check that $\left|N_{G^{8}}\left(x_{4}\right) \cap\left\{y_{6}, y_{7}, y_{8}\right\}\right|=2$-that is, $K_{3,3} \subseteq G^{8}\left[\left\{x_{1}, x_{3}, x_{4}\right\}, Y_{1}\right]$, a contradiction. Hence, $\left|N_{G^{g}}\left(x_{4}\right) \cap\left\{y_{1}, y_{2}\right\}\right|=0$. Therefore, $\left|N_{G^{g}}\left(x_{4}\right) \cap\left\{y_{3}, y_{4}, y_{5}\right\}\right|=2$ and $\left|N_{G^{g}}\left(x_{4}\right) \cap\left\{y_{6}, y_{7}, y_{8}\right\}\right|=2$. W.l.g., we can suppose that $N_{G^{g}}\left(x_{4}\right) \cap Y_{1}=Y_{4}=$ $\left\{y_{3}, y_{4}, y_{6}, y_{7}, y_{9}\right\}$. Since $|D|=3$, so $|E|=4$. W.l.g., suppose that $E=\left\{x_{5}, x_{6}, x_{7}, x_{8}\right\}$. Here, we have a claim as follows:

Claim 8. $\left|N_{G^{g}}\left(y_{9}\right) \cap E\right|=0$.

Proof of Claim 8. By contradiction, suppose that $\left|N_{G^{8}}\left(y_{9}\right) \cap E\right| \neq 0$. Assume that $x_{5} \in$ $N_{G^{g}}\left(y_{9}\right) \cap E$-that is, $x_{5} y_{9} \in E\left(G^{g}\right)$. Since $x_{5} \in E$ and $\left\{x_{2}, x_{3}, x_{4}\right\}=D$, by Claim 3, $\left|N_{G^{g}}\left(x_{5}\right) \cap N_{G^{g}}\left(x_{i}\right)\right|=\left|N_{G^{g}}\left(x_{5}\right) \cap Y_{i}\right|=2$ for $i=2,3,4$. Consider $N_{G^{g}}\left(x_{5}\right) \cap Y_{2}$, assume that $N_{G^{8}}\left(x_{5}\right) \cap Y_{2}=\left\{y^{\prime}, y^{\prime \prime}\right\}$, if $\left\{y^{\prime}, y^{\prime \prime}\right\}=\left\{y_{1}, y_{2}\right\}$, then $\left|N_{G^{g}}\left(x_{5}\right) \cap Y_{4}\right|=1$, a contradiction. Therefore, we can assume that $\left|\left\{y^{\prime}, y^{\prime \prime}\right\} \cap\left\{y_{1}, y_{2}\right\}\right| \leq 1$. If $\left|\left\{y^{\prime}, y^{\prime \prime}\right\} \cap\left\{y_{1}, y_{2}\right\}\right|=0$, then $\left|N_{G^{g}}\left(x_{5}\right) \cap Y_{3}\right|=0$, and if $\left|\left\{y^{\prime}, y^{\prime \prime}\right\} \cap\left\{y_{1}, y_{2}\right\}\right|=1$, then $\left|N_{G^{g}}\left(x_{5}\right) \cap Y_{3}\right| \leq 1$. In any case there exists a vertex of $D$ (say $\left.x^{\prime}\right)$, such that $\left|N_{G^{g}}\left(x_{5}\right) \cap N_{G^{g}}\left(x^{\prime}\right)\right|=1$, a contradiction. So, the assumption does not hold and the claim is true.

Therefore, by Claim 8 , since $\left|N_{G^{8}}\left(y_{9}\right) \cap D\right|=0$, we can say that for any vertex of $N_{G^{g}}\left(y_{9}\right) \cap X \backslash\left\{x_{1}\right\}$ (say $x$ ), $\left|N_{G^{g}}(x) \cap Y_{1}\right| \geq 4$; therefore, by considering $Y_{2}$ and $y_{9}$, as $\left|N_{G^{g}}\left(y_{9}\right) \cap X \backslash\left\{x_{1}\right\}\right|=7$ and $\left|N_{G^{g}}(x) \cap Y_{1}\right| \geq 4$ for each $x \in N_{G^{g}}\left(y_{9}\right) \cap X \backslash\left\{x_{1}\right\}$, the proof is similar to Case 1 , a contradiction.

Therefore, by Cases 1, 2, and 3 the assumption does not hold-that is, $K_{3,3} \subseteq G^{g}$ and the proof of the theorem is complete.

Now, combining Theorems 3-6 yields the proof of Theorem 1.

\section{Discussion}

There are several papers in which the bipartite Ramsey numbers have been studied. In this paper, we proved the conjecture on $B(2,2,3)$, which was proposed in 2015 and states that $B(2,2,3)=17$. We proved this conjecture by a combinatorial argument with no computer calculations. This is significant because computing the exact value of Ramsey numbers is a challenge. To approach the proof of this conjecture, we proved four theorems as follows:

1. Assume that $\left(G^{r}, G^{b}, G^{g}\right)$ is a 3-edge coloring of $K_{17,17}$, where $K_{2,2} \nsubseteq G^{r}, K_{2,2} \nsubseteq G^{b}$ and $K_{3,3} \nsubseteq G^{g}$. Hence, we have:

(a) $\left|E\left(G^{g}\right)\right|=141$. 
(b) $\Delta\left(G^{g}\right)=9$ and $\delta\left(G^{g}\right)=8$.

(c) $D_{G^{g}}(X)=D_{G^{g}}(Y)=(9,9,9,9,9,8,8, \ldots, 8)$.

2. Assume that $\left(G^{r}, G^{b}, G^{g}\right)$ is a 3-edge coloring of $K_{17,17}$, where $K_{2,2} \nsubseteq G^{r}, K_{2,2} \nsubseteq G^{b}$ and $K_{3,3} \nsubseteq G^{g}$. Let $\left|N_{G^{g}}(x)\right|=9$ and $N_{G^{g}}(x)=Y_{1}$, the following results are true:

(a) For each $x \in X \backslash\left\{x_{1}\right\}$, we have $\left|N_{G^{g}}(x) \cap Y_{1}\right| \leq 5$.

(b) Assume that $n=\sum_{i=1}^{i=17}\left|N_{G^{g}}\left(x_{i}\right) \cap Y_{1}\right|$, then $72 \leq n \leq 73$.

3. Assume that $\left(G^{r}, G^{b}, G^{g}\right)$ is a 3-edge coloring of $K=K_{17,17}$, such that $K_{2,2} \nsubseteq G^{r}$, $K_{2,2} \nsubseteq \nsubseteq G^{b}$. Assume that there exists a vertex of $V(K)$ (say $x$ ), such that $\left|N_{G g}(x)\right|=9$. If $\sum_{i=1}^{i=17}\left|N_{G^{g}}\left(x_{i}\right) \cap Y_{1}\right|=73$, where $Y_{1}=N_{G^{g}}(x)$, then $K_{3,3} \subseteq G^{g}$.

4. Assume that $\left(G^{r}, G^{b}, G^{g}\right)$ is a 3-edge coloring of $K=K_{17,17}$, where $K_{2,2} \nsubseteq G^{r}$, $K_{2,2} \nsubseteq \nsubseteq G^{b}$. Assume that there exists a vertex of $V(K)$ (say $x$ ), such that $\left|N_{G^{g}}(x)\right|=9$. If $\sum_{i=1}^{i=17}\left|N_{G^{8}}\left(x_{i}\right) \cap Y_{1}\right|=72$, where $Y_{1}=N_{G^{8}}(x)$, then $K_{3,3} \subseteq G^{g}$.

One might also be able to compute $B\left(n_{1}, \ldots, n_{m}\right)$ for small $i, n_{i}$ like $B(2,3,3,3)$ or $B(3,3,3,3)$ in the future, using the idea of proofs laid out in this paper.

Author Contributions: Conceptualization, Y.R. and M.G.; Formal analysis, Y.R., M.G. and S.S.; Funding acquisition, S.S.; Investigation, Y.R.; Methodology, Y.R., M.G. and S.S.; Resources, Y.R.; Supervision, S.S.; Validation, Y.R.; Writing(original draft), Y.R. and M.G.; Writing(review and editing), M.G. All authors have read and agreed to the published version of the manuscript.

Funding: There was no funding for this work.

Institutional Review Board Statement: Not applicable.

Informed Consent Statement: Not applicable.

Data Availability Statement: This paper focuses on pure graph theory, not involving experiments and data.

Acknowledgments: The authors would like to thank the editors and reviewers.

Conflicts of Interest: The authors declare no conflict of interest.

\section{References}

1. Erdös, P.; Rado, R. A partition calculus in set theory. Bull. Am. Math. Soc. 1956, 62, 427-489. [CrossRef]

2. Kóvari, T.; Sós, V.; Turán, P. On a problem of K. Zarankiewicz. Colloq. Math. 1954, 3, 50-57. [CrossRef]

3. Reiman, I. Über ein Problem von K. Zarankiewicz. Acta Math. Acad. Sci. Hung. 1958, 9, 269-273. [CrossRef]

4. Goddard, W.; Henning, M.A.; Oellermann, O.R. Bipartite Ramsey numbers and Zarankiewicz numbers. Discret. Math. 2000, 219, 85-95. [CrossRef]

5. Beinere, L.W.; Schwenk, A.J. On a bipartite form of the ramsey problem. In Proceedings of the Fifth British Combinatorial Conference, Aberdeen, UK, 14-18 July 1975.

6. Exoo, G. A bipartite Ramsey number. Graphs Comb. 1991, 7, 395-396. [CrossRef]

7. Hattingh, J.H.; Henning, M.A. Star-path bipartite Ramsey numbers. Discret. Math. 1998, 185, 255-258. [CrossRef]

8. Collins, A.F.; Riasanovsky, A.W.; Wallace, J.C.; Radziszowski, S. Zarankiewicz Numbers and Bipartite Ramsey Numbers. J. Algorithms Comput. 2016, 47, 63-78.

9. Dybizbański, J.; Dzido, T.; Radziszowski, S. On some Zarankiewicz numbers and bipartite Ramsey Numbers for Quadrilateral. ARS Comb. 2015, 119, 275-287.

10. Rowshan, Y.; Gholami, M.; Shateyi, S. The Size, Multipartite Ramsey Numbers for nK2 Versus Path-Path and Cycle. Mathematics 2021, 9, 764. [CrossRef]

11. Raeisi, G. Star-path and star-stripe bipartite Ramsey numbers in multicoloring. Trans. Comb. 2015, 4, 37-42.

12. Hatala, I.; Héger, T.; Mattheus, S. New values for the bipartite Ramsey number of the four-cycle versus stars. Discret. Math. 2021, 344, 112320. [CrossRef]

13. Collins, A.F. Bipartite Ramsey Numbers and Zarankiewicz Numbers; Rochester Institute of Technology: Rochester, NY, USA, 2015.

14. Gholami, M.; Rowshan, Y. The bipartite Ramsey numbers $B R\left(C_{-} 8, C_{-}\{2 n\}\right)$. arXiv 2021, arXiv:2108.02630.

15. Bondy, J.A.; Murty, U.S.R. Graph Theory with Applications; Macmillan: London, UK, 1976; Volume 290. 